\title{
THE HESSIAN OF THE CUBIC SURFACE.
}

BY DR. J. T. HUTCHINSON.

(Read before the American Mathematical Society at its Fifth Summer Meeting, Boston, Mass., August 19, 1898.)

\$1. A special form of the Hessian, the coördinates of which are expressible in terms of hyperelliptic functions.

Let $\left[\omega_{i}\right](i=1,2, \cdots, 6)$ be a set of six simultaneous half periods (or briefly, six half periods) forming what might be called a Weber Sextuple, $i$. e., a sextuple having no more than three half periods in common with any Rosenhain sextuple.*

Also let $\theta$ be an even theta function of order four and characteristic zero, vanishing (of the second order) for the six half periods $\left[\omega_{i}\right]$. Every such function can be expressed linearly in terms of four functions of the same kind. Denoting these latter by $x_{1}, x_{2}, x_{3}, x_{4}$, it is evident that between them exists a homogeneous algebraic equation of the fourth degreet

$$
f\left(x_{1}, x_{2}, x_{3}, x_{4}\right)=0 .
$$

Interpreting this equation geometrically as representing a quartic surface, we observe that to the remaining ten half periods correspond ten nodes on the surface.t Also, since there are ten theta functions of the first order

$$
\vartheta_{j} \quad(j=1,2, \cdots, 10)
$$

which vanish for three of the half periods $\left[\omega_{i}\right]$, the ten equations

$$
\vartheta_{j}=0 \quad(j=1,2, \cdots, 10)
$$

represent ten lines on the surface $f . \S$ It is apparent that the ten nodes and the ten lines form the vertices and the edges of a complete pentahedron, and hence the surface $f$ is the Hessian of a cubic. It is not the Hessian of the most general form of the cubic, however, since that depends on

\footnotetext{
* Such a sextuple corresponds, as Weber has shown, to six independent nodes of the Kummer surface, from which the ten remaining nodes can be determined.

†See Humbert, "Théorie générale des surfaces hyperelliptiques," Liouville's Journal, 1893, p. 460.

† Jbid., p. 48.

\& Ibid., p. 44.
} 
four essential constants, while the equation $f=0$ contains only three.

Without attempting any general study of algebraic curves traced on the surface $f$, I will mention some of those of lowest order which appear to have properties of interest.

The function $\vartheta_{j}^{2}$ vanishes of the second order for three of the half periods $\left[\omega_{i}\right]$. A theta function of the second order and characteristic zero which is required to vanish for the three remaining half periods is completely determined. Denoting this function by $\theta_{j}^{(2)}$, the equation

$$
\theta_{j}^{(2)}=0
$$

is the hyperelliptic equation of a conic on the surface $f$. Further, since the product $q_{j}^{2} \theta_{j}^{(2)}$ is an even function of the fourth order and characteristic zero which vanishes for all six of the half periods $\left[\omega_{i}\right]$, and hence is linearly expressible in terms of the coördinate functions $x_{i}$, it follows that the conic $\boldsymbol{\theta}_{j}^{(2)}$ lies in a plane which is tangent to $f$ along the line $\vartheta_{j}$

Suppose $\vartheta_{1}, \vartheta_{2}, \vartheta_{3}, \vartheta_{4}$ represent four lines lying in one of the planes of the pentahedron. The product of these four theta functions is therefore linearly expressible in terms of the coördinate functions, and vanishes to the second order for the six half periods $\left[\omega_{i}\right]$. Hence the function

$$
\theta_{1}^{(2)} \theta_{2}^{(2)} \theta_{3}^{(2)} \theta_{4}^{(2)}
$$

vanishes to the fourth order for the same half periods and is accordingly expressible as a quadratic function of the coördinates. Hence the corresponding four conics lie on a quadric, constituting its complete intersection with $f$. There are five such quadrics.

It is possible to determine a function of the third order $\theta_{j}^{(3)}$ which vanishes of the first order for the three half periods that annul $\vartheta$, and of the second order for the three remaining half periods $\left[\omega_{i}\right]$. The function so determined contains one remaining arbitrary parameter. Hence the equation

$$
\Theta_{j}^{(3)}=0
$$

represents a family of plane cubics lying in the planes having the line $\vartheta_{j}$ for axis. Let $\theta_{1}, \theta_{2}$ denote for brevity two functions of the family (4). It is possible to choose (in two ways) three of the functions occurring in (3), say $\theta_{a}^{(2)}, \theta_{b}^{(2)}, \theta_{c}^{(2)}$, so that $\theta_{a}^{(2)} \theta_{b}^{(2)} \theta_{c}^{(2)} \cdot \theta_{1} \Theta_{2}$ shall be a function vanishing to the sixth order for the half periods $\left[\omega_{i}\right]$, and 
hence expressible as a cubic function of the coördinates. Therefore, any two curves of the same family (4) lie on a cubic surface which meets $f$ elsewhere in three of the conics (3).

If $\vartheta_{i}, \vartheta_{j}, \vartheta_{k}$ represent three lines which meet in a point, the product $\left.\vartheta_{i}^{2} \vartheta_{j}\right\}_{k}$ evideatly vanishes of the second order for two of the half periods $\left[\omega_{i}\right]$, of the third order for two others, and of the first order for the remaining two. Every function $\theta_{j k}^{(t)}$ of the fourth order, and of the same characteristic and odd or even with $\vartheta_{j}{ }_{k}$, vanishes of the first order (or higher) for the four half periods last mentioned above. The arbitrary constants in the general function of this class can be so chosen that the product $\vartheta_{i}^{2} \vartheta_{j} \vartheta_{k} \theta_{j k}^{(4)}$ vanishes of the fourth order for each of the half periods $\left[\omega_{i}\right]$, and hence is expressible as a quadratic function of the coördinates involving one arbitrary parameter. It follows from this that the equation

$$
\Theta_{j k}^{(4)}==0
$$

represents a family of biquadratic curves formed by the intersections with $f$ of a family of cones touching $f$ along one line and intersecting it in two others. As in the case of cubic curves, it is easy to see that any two curves of the same family lie on a cubic surface which touches $f$ along two straight lines.

There are evidently 15 families of such biquadratics corresponding to the 15 characteristics different from zero, and 15 pairs of families of cones containing them.

A second class of biquadratic curves is determined as follows: Suppose $\vartheta_{l}, \vartheta_{m}, \vartheta_{n}, \vartheta_{p}$ correspond to four lines forming a gauche quadrilateral. Let $\theta_{l m n p}^{(4)}$ be a function of the

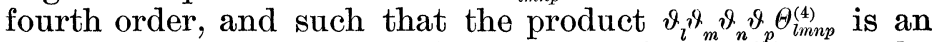
even function of characteristic zero and vanishing to the fourth order for all of the half periods $\left[\omega_{i}\right]$. Then

$$
\Theta_{l m n p}^{(4)}=0
$$

represents a family of $\infty^{1}$ biquadratics having the property that any two curves of the family lie on a cubic surface passing through two conics on $f$.

Each of the 15 families (6) corresponds to one of the fifteen families (5) in such a way that any two curves, one from each of two corresponding families, lie on a quadric. For, a pair of planes can be chosen (in two ways) so as to contain the four lines $\vartheta_{\imath}, \vartheta_{m}, \vartheta_{n}, \vartheta_{p}$. These two planes intersect in a line, say $\vartheta_{i}$, and each intersects $f$ in one other line, say $\vartheta_{j}$ and $\vartheta_{k}$.

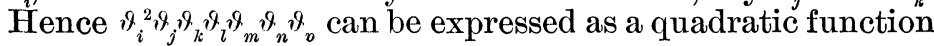


of the coördinates, and accordingly $\theta_{j k}^{(4)} \theta_{l m n p}^{(4)}$ can also be so expressed.

A third class of biquadratics is determined by the even functions $\bar{\theta}_{i j}^{(4)}$, of the fourth order and characteristic zero which vanish of the fourth order for one of the half periods $\left[\omega_{i}\right]$, and of the second order for four other half periods. Let $\left[\omega_{j}\right]$ be the remaining half period. Then, it is possible to find three functions of the form $\vartheta_{i}{ }_{i} \vartheta_{j}{ }^{2}, \vartheta_{k}{ }^{2} \theta_{i}^{(2)}, \theta_{j}^{(2)} \theta_{k}^{(2)}$, each of which vanishes of the fourth order for $\left[\omega_{j}\right]$ and of the second order for all of the other half periods except $\left[\omega_{i}\right]$. The product of $\bar{\theta}_{i j}^{(4)}$ with any one of these three functions is expressible as a quadratic function of the coördinates and hence

$$
\bar{\theta}_{i j}^{(4)}=0
$$

represents an $\infty^{1}$ of biquadratic curves which lie on a family of cones touching $f$ along two lines $\vartheta_{i}$ and $\vartheta_{j}$, on a family of cones touching $f$ along a line $\vartheta_{k}$ and intersecting it in a conic $\Theta_{i}^{(2)}$, and on a family of quadrics intersecting $f$ in two conics $\theta_{j}^{(2)}$ and $\theta_{k}^{(2)}$. It can further be shown that these curves also lie on a family of cubic surfaces which touch $f$ along a line and intersect it in six other lines.

The 30 families of biquadratics (7) are evidently associated two and two, so that two curves, one from each of two associated families, lie on a quadric surface.

I will stop to notice only one other class of curves, a family of $\infty^{3}$ sextics first mentioned by Steiner.*

If $\theta^{(6)}$ be an odd function of the sixth order and characteristic zero, determined so as to vanish of the third order for each of the half periods of the given sextuple, then

$$
\theta^{(6)}=0
$$

represents a family of $\infty^{3}$ sextic curves having the property that any two curves of the family lie on a cubic surface. Since the six edges of a tetrahedron formed by any four planes of the pentahedron is a degenerate sextic of the family (8), it follows that this family of sextics lies on five families of cubic surfaces each family of which intersects $f$ in the six edges of a tetrahedron.

The equation of the hyperelliptic surface $f$ can be derived without difficulty by expanding the coördinate functions in the neighborhood of a pair of half periods and equating to

* Steiner, "Ueber die Flächen dritten Grades," Crelle, vol. 53 (1857), p. 141. 
zero the coefficients of the lowest powers of the infinitesimals occurring in the assumed form for the equation of the Hessian. For the sake of brevity I will employ a notation slightly modified from that used by Humbert in the memoir already quoted. Denoting the four columns

$$
\begin{array}{llll}
1 & 1 & 0 & 0 \\
1, & 0, & 1, & 0
\end{array}
$$

by the digits $1,2,3,4$ respectively, the symbol (12), for example, will be used to denote the theta function of the first order with characteristic $\left[{ }_{10}^{11}\right]$; while, numbering the columns in the reverse order, the symbol 12 will denote the pair of half periods represented by the characteristic $\left[\begin{array}{c}00 \\ 01\end{array}\right]$.

Taking the equation of the Hessian in the form given by equation (9) of the next section, and selecting 11, 22, 33, 21, 31,14 for a sextuple of half periods $\left[\omega_{i}\right]$, we may assume

$$
\begin{aligned}
& w=(11)(12)(31)(32) \\
& x=(13)(24)(32)(41) \\
& y=(11)(21)(13)(23) \\
& z=(21)(31)(24)(34) \\
& u=(12)(23)(34)(41) .
\end{aligned}
$$

The linear relation between these five functions is found to be

$$
u=\alpha w+\beta x+\gamma y+\delta z
$$

where, using the bracket to denote the value of the enclosed function for zero arguments,

$$
\begin{aligned}
& \alpha=-\left[\frac{(11)(42)(33)}{(23)(43)(44)}\right]_{0}, \quad \beta=\left[\frac{(24)(42)(33)}{(22)(34)(43)}\right]_{0} \\
& \gamma=\left[\frac{(11)(33)(24)}{(32)(34)(44)}\right]_{0}, \quad \delta=-\left[\frac{(11)(24)(42)}{(22)(23)(32)}\right]_{0} .
\end{aligned}
$$

The ratios of $a, b, c, d, e$ are found to be

$$
\frac{a}{e}=\alpha, \quad \frac{b}{e}=-\beta, \quad \frac{c}{e}=\gamma, \quad \frac{d}{e}=8 .
$$

\$2. Extension of the Preceding Results to the General Hessian.

The results of the preceding section are easily shown to be true without any modification for the general Hessian. The equation of the Hessian may be written 
(9) $F=a x y z u+b w y z u+c w x z u+d w x y u+e w x y z=0$,

where $w, x, y, z, u$ represent the five planes of the pentahedron. The equation is unchanged in form by the group of 120 permutations made simultaneously on the two sets of letters $w, x, y, z, u$, and $a, b, c, d, e$.

The plane which touches $F$ along the line $w x$ has for equation

$$
b w+a x=0 .
$$

The conic in which this plane meets $F$ also lies on the cone

$$
c z u+d y u+e y z=0 .
$$

Denote this conic by $\{w x\}$. The nine other conics may be denoted in a similar manner by $\{w y\},\{x y\}$, etc.

As was seen in $\$ 1$, the four conics whose symbols have a common letter, say $\{w x\},\{w y\},\{w z\},\{w u\}$, lie on a quadric surface, since (9) can be put in the form

(10) $a^{3} F=(b w+a x)(c w+a y)(d w+a z)(e w+a u)-w^{2} Q_{w}$

where

$$
\begin{gathered}
Q_{w}=b c d e w^{2}+a w(c d e x+b d e y+b c e z+b c d u) \\
+a^{2}(d e x y+c e x z+c d x u+b e y z+b d y u+b c z u) .
\end{gathered}
$$

By means of the permutations

$$
(w x)(a b),(w y)(a c),(w z)(a d),(w u)(a e),
$$

we obtain four other quadrics $Q_{x}, Q_{y}, Q_{z}, Q_{u}$, each of which intersects $F$ in four conics.

Passing now to the family of cubic curves lying in planes through the line $w x$, it can be shown that any two curves of the family lie on a cubic surface passing through the three conics $\{w y\},\{w z\},\{w u\}$. For this purpose multiply both members of (10) by an arbitrary constant $\alpha$, and add and subtract on the right member the term

$$
a^{2} Q_{w}(w+x x)\left(w+x^{\prime} x\right),
$$

$x$ and $x^{\prime}$ being arbitrary constants. We have

where

$$
\alpha a^{3} F=M-a^{2} Q_{w}(w+x x)\left(w+\varkappa^{\prime} x\right)
$$

$$
\begin{gathered}
M=a(b w+a x)(c w+a y)(d w+a z)(e w+a u) \\
+Q_{w}\left[(w+x x)\left(w+x^{\prime} x\right)-\alpha w^{2}\right] .
\end{gathered}
$$

It is now necessary to determine $\alpha$ in such a way that $M$ shall contain the factor $b w+a x$. This being done, the equation takes the form 


$$
\begin{gathered}
(b x-a)\left(b x^{\prime}-a\right) \cdot a^{3} F=(b w+a x) C \\
-a^{2} Q_{w}(w+x x)\left(w+x^{\prime} x\right)
\end{gathered}
$$

where

$$
\begin{gathered}
C=(b x-a)\left(b x^{\prime}-a\right)(c w+a y)(d w+a z)(e w+a u) \\
+Q_{w}\left[a x x^{\prime} x+w\left(a x+a x^{\prime}-b x x^{\prime}\right)\right] .
\end{gathered}
$$

The function $C$ is unchanged for the group of permutations of order six made simultaneously on the two sets of letters $y, z, u ; c, d, e$. Hence there are $\frac{120}{6}=20$ different families of cubic surfaces of the same type as $C$. Two such families of cubic surfaces are associated with each of the ten families of plane cubics, since by the substitution $(w x)(a b)$ equation (11) takes the form

$$
(b x-a)\left(b x^{\prime}-a\right) a^{3} F=(b w+a x) C^{\prime}-b^{2} Q_{x}(w+x x)\left(w+x^{\prime} x\right),
$$

where $C^{\prime}$ is the result of applying the substitution to $C$ after multiplying by a proper constant.

As a special case of the preceding result, we have for $x=x^{\prime}$ the theorem :

With each family of plane cubics is associated two families of cubic surfaces, each of which intersects $F$ in three conics and touches $F$ along the family of cubic curves.

Consider next the family of cones

$$
R=y(e z+d u)-\lambda u z=0
$$

which touches $F$ along the line $z u$ and intersects it in the lines $y z, y u$. It intersects $F$ besides in a family of biquadratics which lie also on the family of cones

$$
S=y(b w+a x)+(c+\lambda) w x=0,
$$

since $F$ can be put in the form

$$
F=w x R+z u S \text {. }
$$

Let $R^{\prime}, S^{\prime}$ denote the functions $R, S$ when $\lambda$ is replaced by a new value $\lambda^{\prime}$. Then the equation

$$
\begin{gathered}
-\lambda^{\prime}(b w+a x) R+\left(c+\lambda^{\prime}\right)(e z+d u) S \\
\equiv-\lambda(b w+a x) R^{\prime}+(c+\lambda)(e z+d u) S^{\prime} \\
\equiv c y(b w+a x)(e z+d u)+\lambda^{\prime} z u(b w+a x) \\
+(c+\lambda)\left(c+\lambda^{\prime}\right) w x(e z+d u)=0
\end{gathered}
$$

represents a cubic surface containing the intersection of $R$ and $S$, and also the intersection of $R^{\prime}$ and $S^{\prime}$, and evidently 
tangent to these cones (and hence tangent to $F^{\prime}$ ) along the lines $w x$ and $z u$.

When $\lambda=\lambda^{\prime}$, we have as a special case of the above: With each of the 15 families of biquadratic curves is associated a family of cubic surfaces of which each surface is inscribed to $F$ along two lines and a biquadratic of the associated family.

Consider the gauche quadrilateral formed by the lines $w u, x z, x u, w z$. The family of quadrics containing these four lines is represented by the equation

$$
N_{\lambda}=w x+\lambda z u=0 .
$$

The most general equation of the quadric containing the lines $y z, y u$, and the conic $\{w x\}$ is

$$
P_{\lambda}=y(b w+a x)-\lambda(c z u+d y u+e y z)=0 .
$$

The intersection of $N_{\lambda}$ and $P_{\lambda}$ lies on $F$, since

$$
F=N_{\lambda} P_{\lambda-1}-N_{\lambda-1} P_{\lambda} \text {. }
$$

Any two biquadratics of the family so determined lie on a cubic surface whose equation has the forms

$$
\begin{aligned}
& c(b w+a x) N_{\lambda}+\left[b w+a x-\lambda^{\prime}(e z+d u)\right] P_{\lambda} \\
\equiv & c(b w+a x) N_{\lambda^{\prime}}+[b w+a x-\lambda(e z+d u)] P_{\lambda^{\prime}} \\
\equiv & (b w+a x)(a x y+b w y+c w x) \\
& -(e z+d u)\left[\lambda x(b w+a x)+\lambda^{\prime} P_{\lambda}\right]=0,
\end{aligned}
$$

showing that the cubic, besides containing the biquadratics whose parameters are $\lambda$ and $\lambda^{\prime}$, also contains the two conics $\{w x\}$ and $\{z u\}$. For $\lambda=\lambda^{\prime}$ we have in the above equation a family of cubic surfaces which touch $F$ along the family of biquadratics.

Each family of the second class of biquadratic curves is associated with a family of the first class so that any two curves being selected, one from each family, a quadric can be found which contains them both. This result appears at once when $F$ takes the form

$$
\begin{gathered}
F=N_{\lambda} R^{\prime}+z u\left[\lambda \lambda^{\prime} z u-\lambda y(d u+e z)+\lambda^{\prime} w x\right. \\
+a x y+b w y+c w x] .
\end{gathered}
$$

The family of cones $K_{\lambda}$ having their vertices at the point wyu and touching $F$ along the lines wy and wu has for equation

$$
K_{\lambda}=\lambda w^{2}+e w y+c w u+a y u=
$$


A second family of cones $K_{\lambda}{ }^{\prime}$ touching $F$ along the line $x z$ and meeting it in the conic $\{y u\}$ is represented by the equation

(13) $K_{\lambda}^{\prime}=\lambda(a x z+b w z+d w x)+(e y+c u)(d x+b z)=0$.

The two cones $K_{\lambda}$ and $K_{\lambda}^{\prime}$ which correspond to the same value of $\lambda$ intersect in a biquadratic lying on $F$ since

$$
a\left(\lambda^{\prime}-\lambda\right) F=K_{\lambda} K_{\lambda^{\prime}}-K_{\lambda^{\prime}} K_{\lambda}{ }^{\prime}
$$

where $K_{\lambda^{\prime}}, K_{\lambda^{\prime}}$ are the functions of equations (12) and (13) with $\lambda^{\prime}$ written in the place of $\lambda$.

Also the family of quadrics

$$
\begin{gathered}
L_{\lambda}=(a x+b w)[\lambda(d w+a z)+d(e y+c u)] \\
+a b(c z u+d y u+e y z)=0
\end{gathered}
$$

which meets $F$ in the two conics $\{w x\}$ and $\{w z\}$ intersect it also in the family of biquadratics defined by $K_{\lambda}$, since

$$
K_{\lambda} L_{\lambda^{\prime}}-K_{\lambda^{\prime}} L_{\lambda}=a^{2}\left(\lambda^{\prime}-\lambda\right) F .
$$

Finally the family of cubic surfaces

$$
\Gamma_{\lambda}=-\lambda w x z+d x y u+b y z u=0
$$

which touches $F$ along the line $x z$, and meets it in the line $w y, w u, x y, z u, y z, u x$, also contains the same family of biquadratics, since

$$
F=w \Gamma_{\lambda}+x y K_{\lambda}
$$

The functions $K_{\lambda}, K_{\lambda}^{\prime}$ are unaltered by the permutation group

1, $(y u)(c e), \quad(x z)(b d), \quad(y u)(x z)(c e)(b d)$.

It therefore follows that there are 30 families of biquadratic curves generated in the same manner as the above mentioned family. Consider in particular the family obtained by applying the permutation $(x y)(z u)(b c)(d e)$ to the preceding equations. Denoting by $K, \overline{K^{\prime}}$, etc., the left members of the new equations and writing $\mu$ in the place of $\lambda$, we have

$$
\begin{gathered}
\bar{K}_{\mu}=\mu w^{2}+d w \cdot c+b w z+a x z \\
{\overline{K^{\prime}}}_{\mu}=\mu(a y u+c w u+e w y)+(d x+b z)(e y+c u) \\
\bar{L}_{\mu}=(a y+c w)[\mu(e w+a u)+e(d x+b z)] \\
+a c(b z u+e x z+d x u) .
\end{gathered}
$$

Any two curves, one from this family and one from the family preceding, lie on a quadric surface. 
For $F$ can be written in the form

where

$$
a F=K_{\lambda} \overline{K_{\mu}}-w^{2} J
$$

$$
\begin{gathered}
J=\lambda \mu w^{2}+\lambda(b w z+d w x+a x z)+\mu(a y u+c w u+e w y) \\
+(b z+d x)(e y+c u) .
\end{gathered}
$$

Also,

$$
a \lambda \mu F=K_{\lambda}^{\prime} \bar{K}_{\mu}^{\prime}-J \cdot(d x+b z)(e y+c u),
$$

and $\quad a(b c d e-a b d \lambda-a c e \mu) \cdot F^{\prime}=L_{\lambda} \bar{L}_{\mu}-Q_{w} J$.

The thirty families of biquadratic curves of this class are associated in pairs in the manner just described.

The five families of cubic surfaces mentioned in the preceding section which intersect $F$ in Steiner's family of sextic curves have for equations the following:

$$
\begin{aligned}
& C_{1}=x e x y z+(x-\lambda) b y z u+(x-\mu) c x z u+(x-\nu) d x y u=0, \\
& C_{2}=\lambda e w y z+(\lambda-x) a y z u+(\lambda-\mu) c w z u+(\lambda-\nu) d w y u=0, \\
& C_{3}=\mu e w x z+(\mu-x) a x z u+(\mu-\lambda) b w z u+(\mu-\nu) d w x u=0, \\
& C_{4}=\nu e w x y+(\nu-x) a x y u+(\nu-\lambda) b w y u+(\nu-\mu) c w x u=0, \\
& C_{5}=x a x y z+\lambda b w y z+\mu c w x z+\nu d w x y=0 .
\end{aligned}
$$

These five functions are connected by the linear relation

$$
a C_{1}+b C_{2}+c C_{3}+d C_{4}=e C_{5} .
$$

That all five cubics intersect $F$ in the same family of sextics is shown by the identities

$$
\begin{array}{cc}
x F=w C_{1}+u C_{5}, & \lambda F=x C_{2}+u C_{5}, \\
\mu F=y C_{3}+u C_{5}, & \nu F=z C_{4}+u C_{5}, \\
\text { etc. }, & \text { etc. }
\end{array}
$$

The family of cubic surfaces

$$
C \equiv x^{\prime} a C_{1}+\lambda^{\prime} b C_{2}+\mu^{\prime} c C_{3}+\nu^{\prime} d C_{4}=0
$$

passes through the sextic $s$ common to $C_{1}, C_{2}, C_{3}, C_{4}$, and cuts out of $F^{\prime}$ a family of $\infty^{3}$ other sextics, $x^{\prime}, \lambda^{\prime}, \mu^{\prime}, \nu^{\prime}$ being arbitrary parameters. These belong to the same family with $s$, since $C$ may also be written

$$
C \equiv \chi_{a} C_{1}^{\prime}+\lambda b C_{2}^{\prime}+\mu d C_{3}^{\prime}+\nu d C_{1}^{\prime},
$$

where $C_{1}^{\prime}, C_{2}^{\prime}, C_{3}^{\prime}, C_{4}^{\prime}$ are the cubics $C_{1}, C_{2}, C_{3}, C_{4}$ with $x, \lambda, \mu, \nu$ replaced by $\varkappa^{\prime}, \lambda^{\prime}, \mu^{\prime}, \nu^{\prime}$, and represent four surfaces 
passing through the sextic $s^{\prime}$ whose parameters are $x^{\prime}, \lambda^{\prime}, \mu^{\prime}, \nu^{\prime}$. Hence: Any two curves of the above mentioned family of sextics lie on a cubic surface.

As a special case we have for $\chi^{\prime}=x, \lambda^{\prime}=\lambda, \mu^{\prime}=\mu, \nu^{\prime}=\nu$ the theorem of Steiner: A cubic surface can be inscribed to the Hessian $F$ along any sextic curve of the family.

Cornell University, Ithaca, N. Y.

\section{ON THE SIMPLE ISOMORPHISMS OF A HAMIL- TONIAN GROUP TO ITSELF.}

BY DR. G. A. MILLER.

(Read before the American Mathematical Society at its Fifth Summer Meeting, Boston, Mass., August 20, 1898.)

IF all the operators of any group $G_{1}$ of a finite order $g_{1}$ are transformed by each of the operators of one of its subgroups they are permuted according to a substitution group that has a 1, $\alpha$ isomorphism to this subgroup. The value of $\alpha$ will, in general, be different for the different subgroups and it will assume its maximum value $\alpha_{1}$ when $G_{1}$ is transformed by all of its operator. The $\alpha_{1}$ operators of $G_{1}$ that are commutative to each one of its operators constitute an abelian characteristic subgroup of $G_{1}$. Hence the factors of composition of $G_{1}$ are the prime factors of $\alpha$, together with the factors of composition of the corresponding quotient group $I_{1}$.

$I_{1}$ is evidently simply isomorphic to the substitution group which is formed by all the permutations of the operators of $G_{1}$ when every operator of $G_{1}$ is transformed by each one of its operators. This substitution group must always be intransitive, since each operator is commutative to itself and hence the substitution group cannot contain any substitution that involves all the elements of the group. When $G_{1}$ is a simple group each of the transitive constituents of this substitution group must be simply isomorphic to $G_{1}$.

From the fact that the given substitution group which is simply isomorphic to $I_{1}$ must be intransitive and does not contain any substitution whose degree is equal to the degree of the group we may easily derive some important 